Bull. Austral. Math. Soc.

VoL. 53 (1996) [271-280]

\title{
A NOTE ON STRONG GEOMETRIC ISOLATION IN 3-ORBITFOLDS
}

\section{DANNY CALEgaRI}

Neuman and Reid describe a 2-cusped hyperbolic 3-orbifold in which the cusps are geometrically isolated. Based on numerical evidence provided by Jeff Weeks' "SnapPea" program, they conjecture that the cusps are strongly geometrically isolated, a fact which we establish here. We also give a parameterisation of the Dehn Stugery Space of this orbifold which has amusing properties.

\section{Geometric Isolation}

Following [3] we make the following definitions:

Given a hyperbolic 3 -orbifold $M$ with cusps $c_{1}, \ldots c_{k}, c_{k+1} \ldots c_{h}$ we say that cusps $1, \ldots, k$ are geometrically isolated from cusps $k+1, \ldots, h$ if any deformation of the hyperbolic structure on $M$ induced by Dehn filling cusps $k+1, \ldots, h$ while keeping cusps $1, \ldots, k$ complete does not change the Euclidean structure at cusps $1, \ldots, k$.

We say that cusps $1, \ldots, k$ are first order isolated from cusps $k+1, \ldots, h$ if the map from the space of deformations induced by Dehn filling cusps $k+1, \ldots, h$ while keeping cusps $1, \ldots, k$ complete to the space of Euclidean structures at cusps $1, \ldots, k$ has zero derivative at the point corresponding to the complete structure.

We say that cusps $1, \ldots, k$ are strongly geometrically isolated from cusps $k+1, \ldots, h$ if for any fixed Dehn filling on cusps $1, \ldots, k$, the geometry of the (possibly filled) cusps $1, \ldots, k$ is unchanged by any Dehn filling on cusps $k+1, \ldots, h$. This can be shown to be equivalent to the fact that $v_{i}\left(u_{1}, u_{2}, \ldots u_{h}\right)$ can be written as a function $v_{i}\left(u_{1}, u_{2}, \ldots, u_{k}\right)$ if and only if $i \leqslant k$ or as a function $v_{i}\left(u_{k+1}, \ldots, u_{h}\right)$ if and only if $i \geqslant k+1$. Here $u_{i}$ and $v_{i}$ are the logarithms of the holonomies of the meridian and longitude respectively of cusp $i$.

It is immediate from these definitions that strong isolation implies geometric isolation, which in turn implies first order isolation.

In [3] it is shown that first order isolation and strong isolation are symmetric conditions in the sets $1, \ldots, k$ and $k+1, \ldots, h$, and that they can be given an analytic definition in terms of the $\Phi$ function defined in [4].

Received 6th June, 1995

I would like to express my gratitude to Walter Neumann and Iain Aitchison with whom I have had many very fruitful conversations regarding the material in this paper. I particularly want to thank Walter Neumann for his generosity in taking the time to make numerous useful remarks on the presentation and details of this material.

Copyright Clearance Centre, Inc. Serial-fee code: 0004-9729/96 \$A2.00+0.00. 


\section{ThE ORBIFOLDS $A^{*}$ AND $A$}

Neumann and Reid in [3] construct a 2-cusped hyperbolic orbifold $A$ with geometrically isolated cusps. Based on numerical evidence, they conjecture that the cusps are strongly isolated. The orbifold $A$ is particularly interesting for two reasons: firstly it is arithmetic, and secondly, all other known examples of manifolds with strongly isolated cusps contain a rigid totally geodesic separating surface which "forces" the isolation. There is evidence that this example does not contain such a surface, based on the fact that its volume is so small. In this paper we find explicit formulae describing how the tetrahedra making up an ideal triangulation of $A^{*}$ are distorted as the hyperbolic structure on $A^{*}$ is deformed which suggests that the existence of such a surface is unlikely.

$A^{*}$ is a 4-cusp link complement in $S^{3} . A$ is obtained from $A^{*}$ by $(2,0)$ Dehn surgery on two of the links. It is a curious fact, which we shall exploit in our calculations, that $A^{*}$ is a double cover of $A$, obtained by a twofold branching of $S^{3}$ over the two filled cusps.
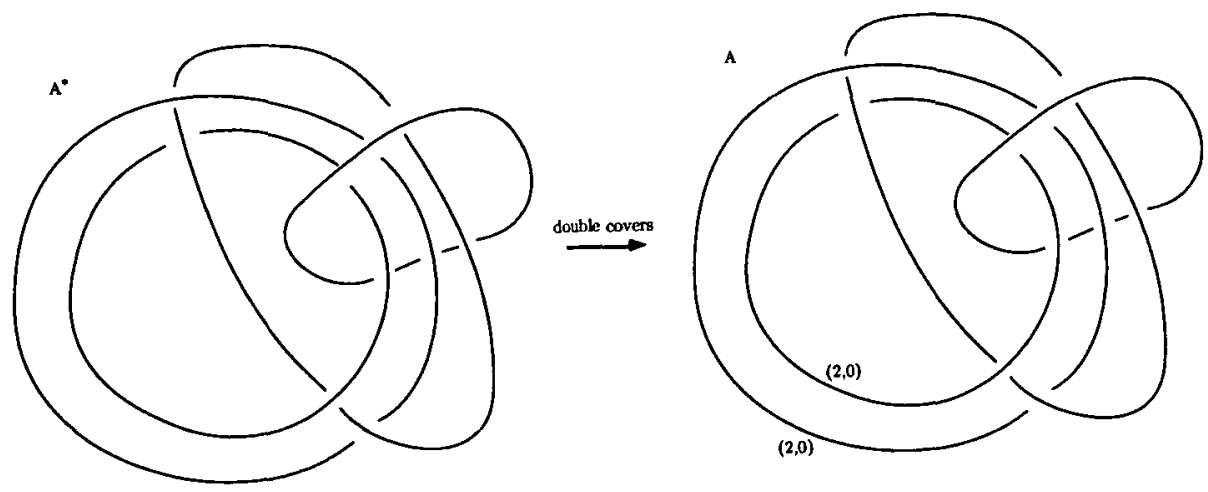

Figure 1. The orbifolds $A^{*}$ and $A$

We can draw $A^{*}$ as a $T^{2} \times(0,1)$ complement. The two removed cusps are linked as in Figure 2. The two cusps in the figure we label as $X$ and $Y . W$ is the cusp $T^{2} \times\{0\}$, and $Z$ the cusp $T^{2} \times\{1\}$. As in [3] we let $u_{i}$ and $v_{i}$ denote the logarithm of the holonomy of $m_{i}$ and $l_{i}$, the meridian and longitude respectively of cusp $i$. Rotating through an angle $\pi / 2$ gives the same link complement after isotopy.

In fact, we can see that $A^{*}$ retains its 4 -fold symmetry even after cusps $X$ and $Y$ are $(m, n)$-filled. Hence the cusp shapes at $T^{2} \times\{0\}$ and $T^{2} \times\{1\}$ are constant. By taking the quotient we see that in $A$ the two cusps are geometrically isolated. Note that this technique can be generalised. Let $M$ be any 4 -fold symmetric $T^{2} \times(0,1) 2$-link 
complement for which there is an orientation-preserving isometry permuting each pair of cusps. By taking the quotient whith respect to this isometry we obtain an orbifold in which one cusp is geometrically isolated from the other. Alternatively, let $M$ be any 4-fold symmetric $T^{2} \times(0,1)$ knot complement. Then the cusp corresponding to this knot is geometrically isolated from the other two cusps. It is not difficult to construct infinitely many examples this way.

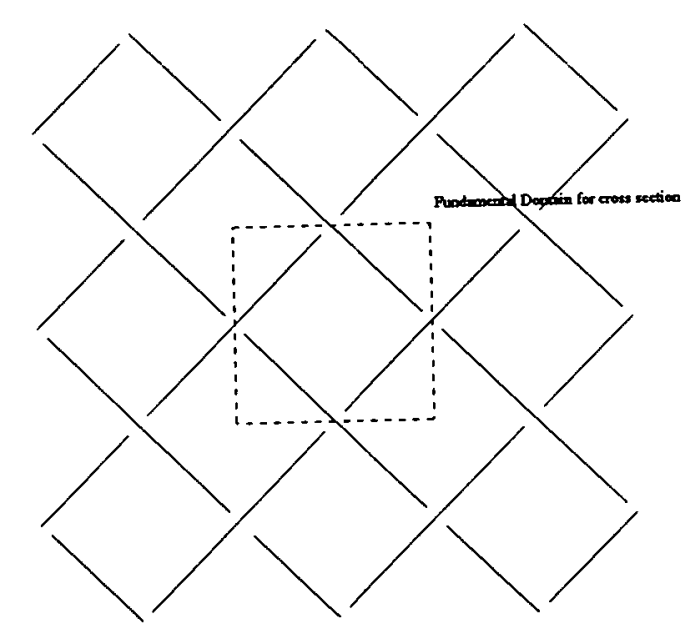

Figure 2. $A^{*}$ as a $T^{2} \times[0,1]$ complement

\section{Strong Isolation of Cusps}

$A^{*}$ has a hyperbolic structure with fundamental domain given by two regular ideal hyperbolic octahedra identified along their boundaries. To see this, note that $A^{*}$ consists of an alternating link complement in $T^{2} \times(0,1)$, and therefore by general properties of alternating links, a fundamental domain for $A^{*}$ can be found by twocolouring the surface on which the link projection lies (in this case a torus), and glueing the regions above and below this surface with a twist of $\pi / 2$ or $-\pi / 2$ according to the colouring (see [1]). The link in $A^{*}$ separates the dividing torus into two squares. The suspension on each of these squares for which the suspension points are cusps $W$ and $Z$ is an octahedron, from which the vertices are removed, since they lie on the cusps. Glueing the top four faces of each octahedron in the obvious manner, the bottom four faces of each are identified with a twist of $\pi$, relative to the top identification. The pattern of identifications is given in Figure 3.

To prove that $A$ is strongly geometrically inolated, it suffices to show that $v_{1}\left(u_{1}, u_{3}\right)$ and $v_{3}\left(u_{1}, u_{3}\right)$ are functions solely of $u_{1}$ and of $u_{3}$ respectively; that is, that we can 

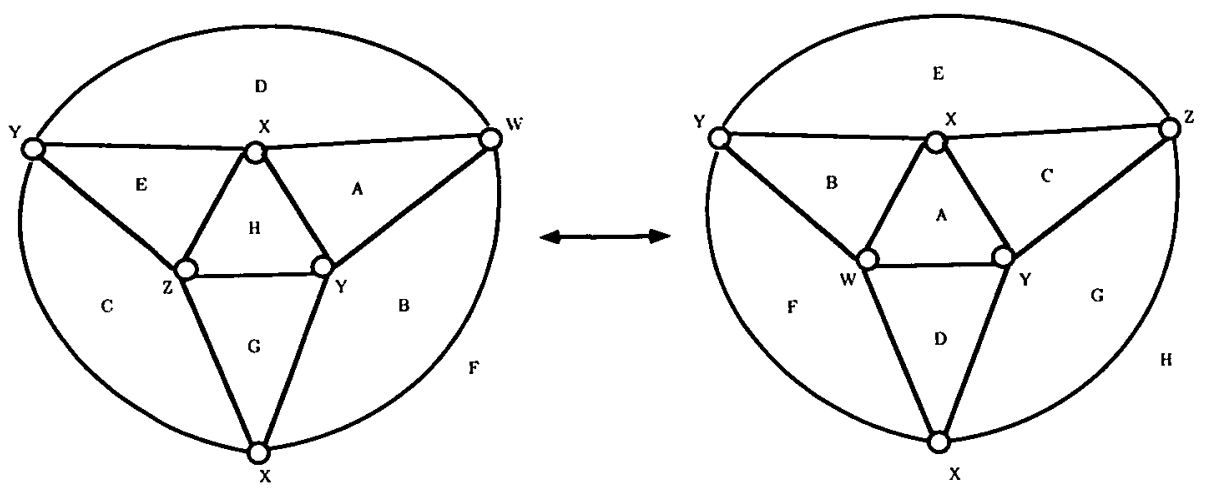

Figure 3. Identifications giving Fundamental Domain for $A^{*}$

define $v_{1}\left(u_{1}\right)$ and $v_{3}\left(u_{3}\right)$.

We subdivide each octahedron into 4 ideal tetrahedra by cutting along the two faces $X Z X W$ and $Y Z Y W$, so that each tetrahedron has vertices $X, Y, Z$ and $W$. We denote the simplex parameters of the four tetrahedra from the first octahedron by $z_{1}, z_{2}, z_{3}, z_{4}$ relative to the central edge, and for the tetrahedra from the second octahedron by $w_{1}, w_{2}, w_{3}, w_{4}$.

The triangulated cusp shapes for the complete structure are given in Figure 4.

In the notation of [3] we have the consistency relations

$$
\begin{gathered}
w_{1} w_{2} w_{3} w_{4}=1 \\
z_{1} z_{2} z_{3} z_{4}=1 \\
w_{1}^{\prime \prime} w_{2}^{\prime} w_{3}^{\prime \prime} w_{4}^{\prime} z_{1}^{\prime} z_{2}^{\prime \prime} z_{3}^{\prime} z_{4}^{\prime \prime}=1 \\
w_{1} w_{3} z_{1} z_{3}=1
\end{gathered}
$$

The holonomies of $l$ and $m$ for the four cusps are

$$
\begin{aligned}
l_{W} & : w_{4}^{\prime \prime} z_{2}^{\prime \prime-1} z_{1}^{\prime-1} w_{1}^{\prime} \\
m_{W}: & z_{2}^{\prime} w_{4}^{\prime-1} w_{3}^{\prime \prime-1} z_{3}^{\prime \prime} \\
l_{Z}: & w_{2}^{\prime \prime} z_{2}^{\prime \prime-1} z_{3}^{\prime-1} w_{1}^{\prime} \\
m_{Z}: & z_{2}^{\prime} w_{2}^{\prime-1} w_{3}^{\prime \prime-1} z_{1}^{\prime \prime} \\
l_{Y}: & z_{3}^{\prime \prime} z_{2}^{\prime \prime-1} w_{2}^{\prime-1} w_{3}^{\prime} \\
m_{Y}: & z_{2}^{\prime} z_{3}^{\prime}{ }^{-1} w_{1}^{\prime \prime} w_{4}^{\prime \prime} \\
l_{X}: & z_{3}^{\prime \prime} z_{4}^{\prime \prime-1} w_{2}^{\prime-1} w_{1}^{\prime} \\
m_{X}: & z_{4}^{\prime} z_{3}^{\prime} i^{-1} w_{3}^{\prime \prime-1} w_{4}^{\prime \prime} .
\end{aligned}
$$


Since we are lifting Dehn surgeries from $A$, we require that

$$
\begin{aligned}
& u_{W}=u_{Z} \\
& u_{Y}=u_{X}
\end{aligned}
$$

from which, and from the Dehn surgery equations, it will follow that

$$
\begin{aligned}
& v_{W}=v_{Z} \\
& v_{Y}=v_{X} .
\end{aligned}
$$

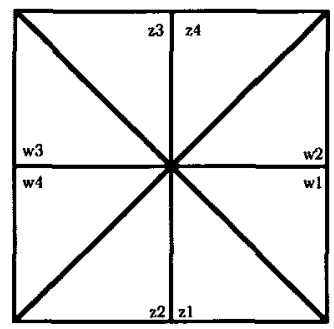

Cusp W

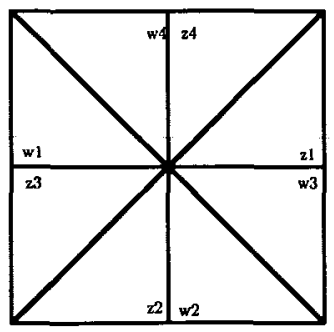

Cusp $Y$

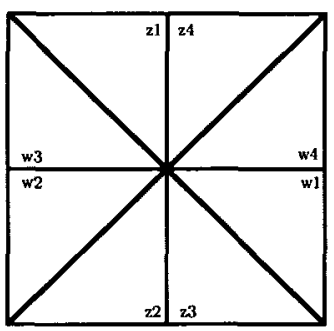

Cusp Z

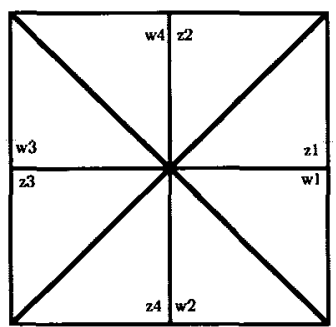

Cusp X

Figure 4. Triangulated Cusp shapes for the Complete Structure

As in [3] we denote $z^{\prime}=(z-1) / z$ and $z^{\prime \prime}=1 /(1-z)$.

Reparameterising, we find that we can write everything in terms of two complex variables, $\alpha$ and $\beta$. We can define the simplex parameters in terms of these two 
variables as follows:

$$
\begin{aligned}
w_{4} & =\frac{\alpha i+\alpha+1-i}{\alpha i-\alpha+1-i}, & z_{1} & =\frac{\alpha+\alpha i}{2-\alpha+\alpha i} \\
w_{2} & =\frac{\alpha i+\alpha-1-i}{\alpha i-\alpha+1+i}, & z_{3} & =\frac{\alpha i+\alpha-2 i}{\alpha i-\alpha} \\
w_{1} & =\frac{2 i-\beta-\beta i}{\beta-\beta i}, & z_{2} & =\frac{i-1-\beta-\beta i}{\beta-\beta i+i-1} \\
w_{3} & =\frac{-\beta-\beta i}{\beta-\beta i-2}, & z_{4} & =\frac{1+i-\beta-\beta i}{\beta-\beta i-i-1} .
\end{aligned}
$$

With these definitions, it can easily be verified that $l_{W}=l_{Z}$ and $m_{W}=m_{Z}$ are functions only of $\beta$, and that $l_{Y}=l_{X}$ and $m_{Y}=m_{X}$ are functions only of $\alpha$; for example, $l_{W}=z_{2}^{\prime \prime-1} w_{1}^{\prime} w_{4}^{\prime \prime} z_{1}^{\prime-1}=z_{2}^{\prime \prime-1} w_{1}^{\prime} / 2$, which is a function only of $\beta$. Explicitly we can calculate

$$
\begin{array}{ll}
l_{W}=\frac{\beta(\beta-i)}{(\beta-1)(\beta-(1+i))}, & m_{W}=\frac{\beta(\beta-1)}{(\beta-i)(\beta-(1+i))} \\
l_{Y}=\frac{\alpha(\alpha-1)}{(\alpha-i)(\alpha-(1+i))}, & m_{Y}=\frac{(\alpha-1)(\alpha-(1+i))}{\alpha(\alpha-i)} .
\end{array}
$$

We therefore have the following:

THEOREM 1. The orbifold $A$ has strongly isolated cusps.

\section{GeOMETRIC DESCRIPTION OF REPARAMETERISATION}

There is a nice geometric definition of the parameterisation in terms of the two complex numbers $\alpha$ and $\beta$, which we now describe.

Given a square $A B C D$ in the complex plane of side length 1 , with vertices at $0,1,1+i, i$, we choose a point $O$ at the complex number $\alpha$. We construct the points $R, S, T$ and $U$ so that the triangles $A S O, B T O, C U O$ and $D R O$ are similar with angles $\pi / 4, \pi / 2$ and $\pi / 4$, as illustrated in Figure 5 . Then it is an easy calculation to see that as complex numbers, $T=R+1$ and $S=U+i$. Therefore the octagon $A S B T C U D R$ tiles the complex plane.

The four triangles $D U O, C T O, B S O$ and $A R O$ are precisely (up to similarity) the horoball sections of the simplices $z_{2}, w_{3}, z_{4}$ and $w_{1}$ respectively after Dehn filling cusps $Z$ and $W$.

There is a similar, though "mirror reversed" picture describing how $w_{2}, w_{4}, z_{1}$ and $z_{3}$ are determined as a function of $\alpha$. From the fact that this octagon tiles the plane, the consistency relations $w_{1} w_{3}=z_{2} z_{4}=-1$ and $w_{1}^{\prime \prime} w_{3}^{\prime \prime} z_{2}^{\prime \prime} z_{4}^{\prime \prime}=-1 / 4$ are geometrically 


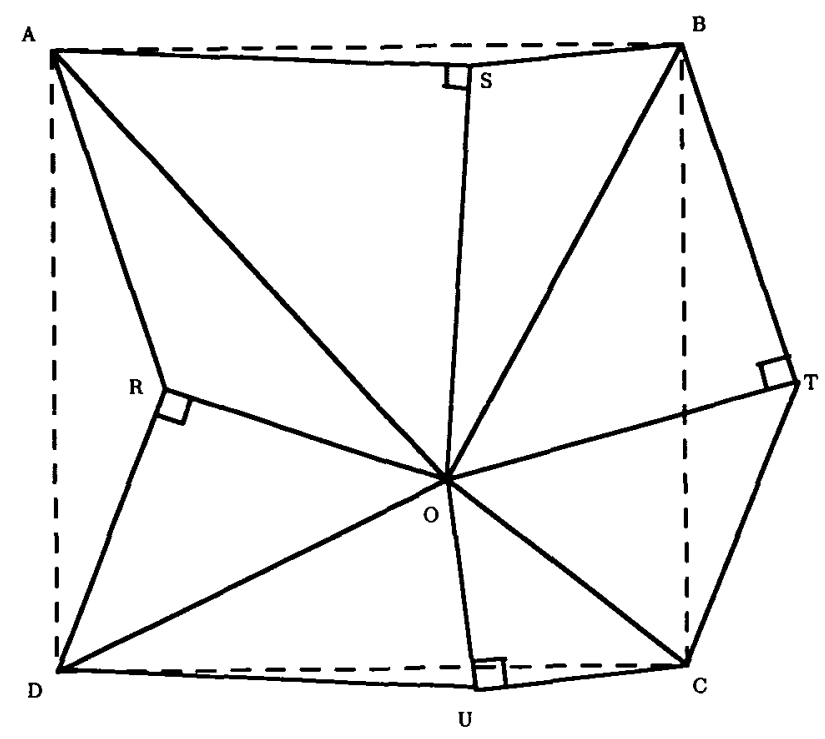

Figure 5. Geometric description of reparameterisation

obvious. From the mirror-reversed picture, the consistency relations $w_{2} w_{4}=z_{1} z_{3}=-1$ and $w_{2}^{\prime} w_{4}^{\prime} z_{1}^{\prime} z_{3}^{\prime}=-4$ can similarly be deduced.

A relation of the form $w_{4}^{\prime \prime} z_{1}^{\prime-1}=1 / 2$ is equivalent to the geometric fact that the orientation preserving similarity taking $A O$ to $O S$ takes $R$ to the midpoint of $O B$. From simple relations such as these, the independence of $l_{W}$ and $m_{W}$ from $\alpha$ and of $l_{Y}$ and $m_{Y}$ from $\beta$ can be seen.

\section{The Dehn Surgery Parameter Space}

There is an interesting relationship between the parameterisation of Dehn Surgery Parameter Space by real pairs $p_{i}, q_{i}: i=1,2$, and by $\alpha, \beta$. In particular, we have the following:

THEOREM 2. The circle

$$
|\beta-1 / 2-i / 2|=1 / \sqrt{2}
$$

corresponds to the values of $(p, q)$ lying on the square with vertices

$$
(-2,-2) ;(-2,2) ;(2,-2) ;(2,2) \text {. }
$$

Notice of course that a corresponding fact holds for $p_{1}, q_{1}$ and $\alpha$. 
Proof: The circle $|\beta-1 / 2-i / 2|=1 / \sqrt{2}$ passes through the square in the complex plane with vertices at $0,1, i, 1+i$. Therefore, by the description in Section 4 , it corresponds precisely to the values of $\beta$ for which four of the simplices are degenerate. That is to say, the simplex parameters $w_{1}, w_{3}, z_{2}, z_{4}$ are real. By symmetry, we need only consider the case that $\beta$ lies on a fixed "quadrant" of the circle, for the sake of argument, the arc between $D$ and $C$ in Figure 5 .

However we know that

$$
\begin{aligned}
& v_{W}=\log \left(1-z_{2}\right)+\log \left(w_{1}-1\right)-\log \left(w_{1}\right)-\log (2) \\
& u_{W}=\log \left(z_{2}-1\right)+\log \left(1-w_{3}\right)-\log \left(z_{2}\right)-\log (2)
\end{aligned}
$$

and moreover, that

$$
p_{2} u_{W}+q_{2} v_{W}=2 \pi i .
$$

But then, by inspecting Figure 5, it can be seen that for $\beta$ taking values on this arc, $w_{1} \leqslant-1, z_{2}>1$ and $0<w_{3}<1$, so that $\Im\left(v_{W}\right), \Im\left(u_{W}\right)$ must take the values of $\pi, 0$ respectively. Hence, $q_{2}=2$. It is a simple matter to check that $\left|\Re\left(v_{W}\right)\right| \leqslant\left|\Re\left(u_{W}\right)\right|$, and therefore that $\left|p_{2}\right| \leqslant 2$.

By a continuity argument, it can be seen that vertices $C$ and $D$ correspond to the points $(-2,2)$ and $(2,2)$ in $p_{2}, q_{2}$ space, and the proof follows.

Corollary 1. $\operatorname{Vol}\left(A_{\left(p_{2}, q_{2}\right)}\right)=\operatorname{Vol}(A) / 2$ for $\left(p_{2}, q_{2}\right)$ taking values on the square with vertices as in Theorem 2 .

By a similar argument we can show:

THEOREM 3. The "circle"

$$
|\beta|=\infty
$$

corresponds to the values of $\left(p_{2}, q_{2}\right)$ lying on the square with vertices

$$
(-1,-1) ;(-1,1) ;(1,-1) ;(1,1) \text {. }
$$

For the sake of argument, the infinite circle can be understood as a "limit" of larger and larger circles, in the Hausdorff topology on the standard compactification $\overline{\mathbf{C}}$.

Proof: It is easy to see that as $\beta \rightarrow \infty$, the simplex parameters

$$
z_{2}, z_{4}, w_{1}, w_{3} \rightarrow-i
$$

(that is to say, their orientation and volume is negative). This implies that each of $v_{W}, u_{W}$ approaches the value of 0 or $\pm 2 \pi i$ according to which branch of the logarithm is taken. This depends on which homotopy path $\beta$ takes from $1 / 2+i / 2$ to $\infty$ in the 
4-punctured sphere $\overline{\mathbf{C}}-\{0,1, i, 1+i\}$. We restrict the values of $\beta$ for the moment to the simply connected domain consisting of $\mathbf{C}$ minus the four infinite rays emanating from the four corners of the square. The appropriate branch of the logarithm can then be determined by observing how the simplex parameters deform as $\beta \rightarrow \infty$ and then requiring that $u_{W}, v_{W}$ be continuous. It remains to check that $\left|p_{2}\right|,\left|q_{2}\right| \leqslant 1$ in the limit. Without loss of generality, we choose $\beta$ with $\Re(\beta)>\Im(\beta)>>0$. Then $p_{2} \rightarrow 1$, by the argument above. It suffices to check that $\left|\Re\left(v_{W}\right)\right| \geqslant\left|\Re\left(u_{W}\right)\right|$ as $\beta \rightarrow \infty$. But by the parameterisation in terms of $\beta$, it is immediate that $\left|l_{W}\right|>\left|m_{W}\right|>1$. The case for other large absolute values of $\beta$ follows by symmetry, and a continuity argument for the cases that $|\Re(\beta)|=|\Im(\beta)|$. The proof follows.

One must be wary about assuming that geometric structures corresponding to the case that $\left(p_{2}, q_{2}\right)$ lie in the interior of the square with vertices as in Theorem 2 exist.

For the case that $\left(p_{2}, q_{2}\right)$ lie on this square, four of the tetrahedra are flat and disjoint except at the vertices. The other four tetrahedra can be glued to the flat four, to produce an incomplete hyperbolic manifold, by the consistency equations. The fact that each flat tetrahedra has a (geometric) bicollared neighbourhood in the manifold so obtained not intersecting any of the other four tetrahedra implies that one can 'pump some air' into each of the four flat tetrahedra without changing the homeomorphism type of the manifold. That is to say, the incomplete manifold so obtained is homeomorphic to $A^{*}$, and the complete manifold obtained by performing geometric Dehn surgery is homeomorphic to a manifold obtained by topological Dehn surgery on $A^{*}$. Note by a theorem proved in [2] one can find geometric structures corresponding to $\left(p_{2}, q_{2}\right)$ in an open neighbourhood of this square - that is to say, including some cases in which four of the tetrahedra are negatively oriented. Notice that as $\beta$ approaches one of $0,1, i, 1+i$ that one of the $(1,1, \sqrt{2})$ triangles in a horoball section gets arbitrarily small with respect to the other three. This is to say, the length of the filled geodesic in a complete structure (if one were to exist) goes to infinity. This suggests that the orbifolds $A_{( \pm 2, \pm 2)}$ contain incompressible tori or annuli.

\section{REFERENCES}

[1] I.R. Aitchison and J.H. Rubinstein, 'Canonical surgery on alternating link diagrams', in Knots '90 Proceedings of the International Conference on Knot Theory and Related Topics, Osaka, August 15-19, 1990, (Akio Kawauchi, Editor), pp. 543-558.

[2] C.D. Hodgson, Degeneration and regeneration of geometric structures on three-manifolds, PhD thesis (Princeton University, 1986).

[3] W.D. Neumann and A.W. Reid, 'Rigidity of cusps in deformations of hyperbolic 3- orbifolds', Math. Ann. 295 (1993), 223-237.

[4] W.D. Neumann and D. Zagier, 'Volumes of hyperbolic three-manifolds', Topology 24 (1985), 307-332. 
Department of Mathematics The University of Melbourne Parkville Vic 3052

Australia 